\title{
Editorial
}

\section{How to test for antineutrophil cytoplasmic antibodies- evidence based immunology?}

Antineutrophil cytoplasmic antibodies (ANCA) are now well established as a useful diagnostic test in the investigation of vasculitis and necrotising glomerulonephritis. The initial assay was an immunofluorescent assay using human neutrophils as a target. By a serendipitous quirk, fixation with ethanol allows two discrete patterns to be identified: C-ANCA and P(perinuclear)-ANCA. The major antigenic specificities are proteinase-3 (C-ANCA) and myeloperoxidase (P-ANCA).* Other neutrophil enzymes have been identified as target antigens, but the association with diseases for these is not so clearly established. The identification of the major target antigens has led to the development of solid phase assays against the relevant antigens. The question now is what is the most appropriate strategy for testing for ANCA, meeting the criteria of accuracy, reproducibility, and cost-effectiveness. Several papers recently submitted to the Journal help clarify a number of important issues. The internationally accepted gold standard remains indirect immunofluorescence on human neutrophils fixed with ethanol. ${ }^{1}$ Experienced microscopists can readily distinguish the major patterns from atypical patterns (sometimes found in inflammatory bowel disease). A paper by Savige et al provides an excellent reference work on the major patterns of fluorescence seen on neutrophils, correlated with solid phase assays. ${ }^{2}$ They draw attention to the important point that identification of C-ANCA must follow specified criteria, and that it is easy to misidentify atypical C-ANCA as true C-ANCA. A further paper from some of the same investigators ${ }^{3}$ confirms the importance of correct identification of the pattern in terms of disease correlation and correlation with the presence of PR3 antibodies. Thus careful microscopy can accurately identify sera containing PR3 antibodies. Unfortunately, in the United Kingdom ${ }^{4}$ and in the Australasian EQA schemes, ${ }^{2}$ many laboratories fail to distinguish atypical C-ANCA from true PR3+ C-ANCA, thus misreporting samples and potentially misleading clinicians.

Difficulties are also experienced in distinguishing P-ANCA from granulocyte specific or other antinuclear antibodies. It has been suggested that the additional use of formalin fixed neutrophils helps, as $\mathrm{MPO}+$ sera are supposed to give a cytoplasmic staining pattern. This technique has been widely adopted in the United Kingdom, based a Broadsheet published in this Journal. ${ }^{5}$ Unfortunately, at the time this technique had never been formally evaluated in the international workshops and there were no adequate data confirming the technique to be useful. There is no standardised method for the use of formalin fixed neutrophils, and our own previously published data ${ }^{6}$ and more recent extended data presented at the last International ANCA Workshop in Birmingham confirm that this technique is not reproducible. Informal discussions with leading international figures in the field at this meeting confirmed that many had tried formalin fixation but had also found it to

* Editorial note: the new international nomenclature assigns capitals for C (classical) and P (perinuclear) ANCA. be unreliable, although it is regrettable that these "negative data" have not been placed in the public domain. Why then is this technique so popular, when there is no supporting evidence and the published and unpublished expert view is that the technique is misleading?

This brings one straight to the role of solid phase assays for ANCA detection. One might assume that these immediately have a significant advantage: purified antigen means no difficulty in identifying PR3 and MPO reactive antibodies no matter what other antibodies are present. The major disadvantage is cost, particularly if two separate assays have to be run together to obtain the same information as can be obtained from one cytospin of neutrophils. How will testing develop when commercial assays are available for other neutrophil enzymes? Will we end up with a similar muddle as with testing for antibodies to extractable nuclear antigens, where there are so many kits available and all giving different answers? With no evidence based consensus on which methods give clinically relevant answers, the EQA performance is uninterpretable. Our own data (Chowdhury Z, Broomhead V, and Spickett G, in preparation) show that while most solid phase assays perform reasonably robustly, there may be both false positives and false negatives, and patients with dual positivity for both MPO and PR3. We also have no knowledge yet about how the numeric absorbance values for the ELISA assays correlates with titres obtained by immunofluorescence: are they a more accurate predictor of clinical relapse? Before any more assays are introduced, evaluation of the place in testing strategies of the existing assays needs to be completed.

A recent international consensus has been agreed on ANCA detection, which sets minimal and optimum strategies based on available fluorescent and solid phase assays. ${ }^{7}$ In summary, the minimum strategy for samples without ANA present is for immunofluorescence followed by solid phase MPO and PR3 assays. The authors comment that $10 \%$ of sera will only be positive by immunofluorescence and $5 \%$ only positive in solid phase assays. Where an ANA or other interfering antibody is present, solid phase assays should be used in place of immunofluorescence as the test of first selection. It is suggested that where the ELISA is positive the ELISA units should be reported rather than the titre of immunofluorescencedespite the fact that there is no agreed international standard, all the kits have different arbitrary units at present, and the applicability of changing ELISA units in predicting relapse has not been confirmed, as it has for IIF titres. It should be noted that formalin fixation has no role in this agreed international consensus.

Solid phase assays should not replace immunofluorescence as the initial screen, except in the circumstance of interfering ANA - in expert hands much more information can be obtained from a single ethanol fixed cytospin of neutrophils than a battery of solid phase assays, and certainly this is more cost-effective strategy. This is of 
course dependent on having properly trained staff who can distinguish the patterns correctly, and this is clearly not the case at present. However, lack of proper skills should not be used as an excuse to inflate the cost of testing by using vastly more expensive kits, which have yet to be fully proven. Solid phase assays can be used more costeffectively as confirmatory tests, in a non-quantitative way (until evidence is produced to the contrary), thus reducing the laboratory costs substantially. The problem of antinuclear antibodies can be addressed simply through appropriate screening for ANA with either standard rodent liver sections or HEp-2 cells. There is no place in this schema for formalin fixation.

The lack of appropriate microscopy skills needs to be addressed by reducing the number of laboratories doing the test-so that the throughput in the remainder is increased, so providing sufficient numbers to maintain expertise-and through appropriate educational workshops. In the meantime all those reading ANCA immunofluorescence should ask themselves how good they are at distinguishing typical and atypical ANCAs and, when they detect them, whether they are giving the appropriate information to clinicians. After all, only 11 of 134 laboratories in the United Kingdom appear to be able to do this at the moment, ${ }^{4}$ although to be entirely fair, the current NEQAS return form for ANCA does not at present give scope for reporting atypical patterns easily; some laboratories may therefore have noted the abnormality but still reported in the C-ANCA box. As NEQAS returns are supposed to reflect clinical practice, commenting on atypical patterns is required.
The answer to problems in ANCA detection, however, is not to reach for the formalin bottle or the expensive ELISA kit but to concentrate on basic skills: proper use of fluorescent conjugates, good quality fluorescent microscopes, correctly aligned, and of course experienced personnel, appropriately trained and supervised. These skills should then be used to collect appropriate evidence on which to devise robust and cost-effective testing strategies. Then and only then will the clinicians have confidence in the results provided by diagnostic laboratories.

G P SPICKETT

V BROOMHEAD

Regional Department of Immunology, Royal Victoria Infirmary, Newcastle upon Tyne NE1 4LP, UK

1 Wiik A. Delineation of a standard procedure for indirect immunofluorescence detection of ANCA. Acta Pathol Microbiol Immunol Scand 1989; 97(suppl 6):12-13.

2 Savige JA, Paspaliaris B, Silvestrini R, et al. A review of immunofluorescent patterns associated with antineutrophil cytoplasmic antibodies (ANCA) and their differentiation from other antibodies. F Clin Pathol 1998;51:56875 .

3 Wong RCW, Silvestrini RA, Savige JA, et al. Diagnostic value of classical and atypical cytoplasmic ANCA immunofluorescence patterns. $f$ Clin Pathol 1999;52:124-8.

4 Jolles S, Deacock S, Turnbull W, et al. Atypical cANCA following high dose intravenous immunoglobulin. $\mathcal{F}$ Clin Pathol (in press).

5 Lock RJ. Detection of autoantibodies to neutrophil cytoplasmic antigens. $\mathcal{F}$ Clin Pathol 1994;47:4-8.

6 Spickett GP, Broomhead V. Formalin fixation and patterns of antineutrophil cytoplasmic antibodies. F Clin Pathol 1995;48:89-90.

7 Savige J, Gillis D, Davies D, et al. International consensus statement on testing and reporting of antineutrophil cytoplasmic antibodies (ANCA). Am $\mathcal{F}$ Clin Pathol (in press). 